\title{
SOLVABLE NUMBER FIELD EXTENSIONS OF BOUNDED ROOT DISCRIMINANT
}

\author{
JONAH LESHIN \\ (Communicated by Matthew A. Papanikolas)
}

\begin{abstract}
Let $K$ be a number field and $d_{K}$ the absolute value of the discriminant of $K / \mathbb{Q}$. We consider the root discriminant $d_{L}^{\frac{1}{[L: \mathbb{Q}]}}$ of extensions $L / K$. We show that for any $N>0$ and any positive integer $n$, the set of length $n$ solvable extensions of $K$ with root discriminant less than $N$ is finite. The result is motivated by the study of class field towers.
\end{abstract}

\section{INTRODUCTION}

Let $K$ be a number field with Hilbert class field $H_{K}$. Set $K=K^{(0)}$ and define $K^{(i)}:=H_{K^{(i-1)}}$ for all $i \geq 1$. Let $K^{(\infty)}=\bigcup_{i} K^{(i)}$. We say $F$ has finite class field tower if $K^{(\infty)}$ is a finite extension of $\mathbb{Q}$, i.e., if the $K^{(n)}$ stabilize for large $n$. If $K^{(\infty)}$ is infinite over $\mathbb{Q}, K$ has infinite class field tower. The main theorem on class field towers is that of Golod and Shafarevich, who proved in 1964 that number fields with infinite class field tower exist 8 .

We define the root discriminant of $K$ to be

$$
r d(K):=d_{K}^{\frac{1}{[K: \mathbb{Q}]}} .
$$

Given a tower of number fields $L / K / F$, we have the following equality of ideals of F:

$$
d_{L / F}=N_{K / F}\left(d_{L / K}\right)\left(d_{K / F}\right)^{[L: K]},
$$

where $d_{L / F}$ denotes the relative discriminant. (With this notation, $d_{F}$ denotes the absolute value of $d_{F / \mathbb{Q}}$.) It follows from (1) that if $L$ is an extension of $K$, then $r d(K) \leq r d(L)$, with equality if and only if $d_{L / K}=1$; i.e., $L / K$ is unramified at all finite primes. Thus if $K$ has infinite class field tower, then all fields $K^{(i)}$ have the same root discriminant; in particular, the set

$$
Z_{N, K}:=\{L: L / \mathbb{Q} \text { finite, } L \supseteq K, \text { and } \operatorname{rd}(L) \leq N\}
$$

is infinite for $N \geq \operatorname{rd}(K)$.

From discriminant bounds originally due to Odlyzko and others, there exist only finitely many number fields $K$ with $r d(K) \leq \Omega:=4 \pi e^{\gamma} \approx 22$, where $\gamma$ is Euler's constant, and this number can be improved to $2 \Omega$ if we assume the Generalized Riemann Hypothesis [2]. It follows that a number field with infinite class field tower must have root discriminant larger than $\Omega$. Martinet has constructed a number field with infinite class field tower and root discriminant $\approx 92.4$. Using

Received by the editors December 12, 2011.

2010 Mathematics Subject Classification. Primary 11R20, 11R29; Secondary 11-XX.

(C) 2013 American Mathematical Society Reverts to public domain 28 years from publication 
tamely ramified class field towers, Hajir and Maire gave an example of a number field $K$ with $Z_{82.2, K}$ infinite [2].

We will not be concerned with specific values of root discriminants but rather with the following question: fix a number field $K$ and an arbitrary (large) real number $N>0$. How can infinite subsets of $Z_{N, K}$ arise? For example, fix a positive integer $n$. If $N$ were large enough, could the set

$$
\{L: L / \mathbb{Q} \text { finite, } L / K \text { solvable length } n, \text { and } \operatorname{rd}(L) \leq N\}
$$

be infinite?

The answer to the question is no, and this is the main theorem of the paper.

Theorem 1. Fix a number field $K$, a positive integer $n$, and a positive real number $N$. The set

$$
Y_{n, N, K}:=\{L: L / \mathbb{Q} \text { finite, } L / K \text { solvable length } n \text {, and } r d(L) \leq N\}
$$

is finite.

Remark 1. - Taking $n=1$ gives finiteness for abelian extensions. The general solvable case follows by induction from the $n=1$ case, and the proof of the $n=1$ case occupies the bulk of the paper. We set $Y_{N, K}:=Y_{1, N, K}$.

- Rather than considering the root discriminant of extensions $L$ of $K$, we could equivalently consider the quantity $\left(N_{K / \mathbb{Q}} d_{L / K}\right)^{1 /[L: K]}$. This is evident by (1).

- Odlyzko mentions in [7] that $Y_{N, \mathbb{Q}}$ is known to be finite for any $N$, but he does not give a proof.

From here on, all field extensions are assumed to be finite unless otherwise stated.

\section{DiscriminANTS AND RAMIFICATION GROUPS}

Let $L / K$ be a Galois extension of local fields, with $K$ a finite extension of $\mathbb{Q}_{p}$. In [3], Hilbert gives the following formula for the relative different $D_{L / K}$ in terms of the ramification groups $G_{i}$ of $L / K$ :

$$
v_{L}\left(D_{L / K}\right)=\sum_{i=0}^{\infty}\left(\left|G_{i}\right|-1\right),
$$

where $v_{L}$ denotes the normalized $\mathfrak{P}$-adic valuation of a fractional ideal of $O_{L}, \mathfrak{P}$ the unique maximal ideal of $O_{L}$. If $L / K$ is now a Galois extension of global fields with $\mathfrak{P}$ a prime of $L$ lying above a prime $\mathfrak{p}$ of $K$, then

$$
v_{\mathfrak{p}}\left(d_{L / K}\right)=g f \sum_{i=0}^{\infty}\left(\left|G_{i}\right|-1\right),
$$

where $G_{i}$ are the ramification groups of $\mathfrak{P} / \mathfrak{p}, g$ is the number of primes of $L$ above $\mathfrak{p}$, and $f$ is the residue degree of $\mathfrak{P} / \mathfrak{p}$. So for a Galois extension $K / \mathbb{Q}$, we obtain

$$
v_{p}(r d(K))=\frac{1}{\left|G_{0}\right|} \sum_{i=0}^{\infty}\left(\left|G_{i}\right|-1\right) .
$$

Note that if we define the root discriminant of a finite extension $K_{\mathfrak{p}}$ of $\mathbb{Q}_{p}$ to be

$$
r d\left(K_{\mathfrak{p}}\right)=p^{\frac{v_{\mathbb{Q}_{p}}\left(d_{K_{\mathfrak{p}} / \mathbb{Q}_{p}}\right)}{\left[K_{\mathfrak{p}}: \mathbb{Q}_{p}\right]}},
$$

then $p^{v_{p}(r d(K))}=r d\left(K_{\mathfrak{p}}\right)$. 


\section{Proof of Theorem 1}

Fix a number field $K$ and a real number $N>0$. Our first goal is to show that the set

$$
X_{N, K}:=\{L: L / K \text { abelian, } L / \mathbb{Q} \text { Galois, and } \operatorname{rd}(L) \leq N\}
$$

is finite in the case when $K / \mathbb{Q}$ is Galois.

If $E / F$ is a Galois extension of number fields ramified at a prime $\mathfrak{p}$ of $F$ with $e=e_{E}(\mathfrak{p})$, then by (2), $\mathfrak{p}^{(e-1) f g} \mid d_{L / K}$. It follows that if $L$ is a number field Galois over $\mathbb{Q}$ with $r d(L) \leq N$, then $L / \mathbb{Q}$ cannot ramify at any rational prime $p$ with $p>\sqrt{N}$.

Let $S$ be the union of the real places of $K$ and the set of primes of $K$ lying above the rational primes $p$ with $p \leq \sqrt{N}$. Suppose that $X_{N, K}$ is infinite. Then there exists an increasing sequence of natural numbers $n_{l}$ such that $\left[L_{n_{l}}: K\right]=n_{l}$ and $L_{n_{l}} \in X_{N, K}$. For a fixed positive integer $m$, the maximal abelian extension of $K$ of exponent $m$ that is unramified outside $S$ is finite [11, Chapter 8.1, Prop. 1.6]. Thus we may assume that $L_{n_{l}} / K$ is cyclic for each $l$. We deal with the following two cases separately:

Case I. For every $\mathfrak{p} \in S$, limsup $e_{L_{n_{l}}}(\mathfrak{p})<\infty$ (the limsup being indexed by $\left.l\right)$.

To ease notation, write $L_{l}$ for $L_{n_{l}}$. Write $S=\left\{\mathfrak{p}_{j}\right\}$. Let $\mathfrak{f}_{l}=\mathfrak{f}\left(L_{l} / K\right)$ be the conductor of $L_{l} / K$. As $l$ gets arbitrarily large, there exists $j$ such that the power $a_{j}$ of $\mathfrak{p}_{j}$ dividing $\mathfrak{f}_{l}$ gets sufficiently large. This is because $L_{l} / K$ can be ramified only at the primes in $S$, so its conductor is divisible by only these primes, which means $L_{l}$ is contained in the ray class field of $K$ modulo the product of the infinite real places of $K$ and a power $m_{l}$ of the product of the finite primes of $K$ ramifying in $L_{l} / K$. As $\left[L_{l}: K\right]$ increases, the minimal such $m_{l}$ increases, which by definition of the ray class field implies that $a_{j}$ increases for some $j$.

Set $\mathfrak{p}=\mathfrak{p}_{j}$ and $a=a_{j}$. Let $\mathfrak{P}_{l}$ be a prime of $L_{l}$ lying above $\mathfrak{p}$. Write $\hat{L}_{l}$ for $L_{\mathfrak{P}_{l}}$, and to keep notation consistent write $\hat{K}$ for $K_{\mathfrak{p}}$. Define the group of $n$-units $U^{n}$ of $\hat{K}$ to be the group of units of $O_{\hat{K}}$ that are congruent to $1\left(\bmod \mathfrak{p}^{n}\right)$. The $\mathfrak{p}$-contribution to $\mathfrak{f}_{l}$ is the conductor of the abelian extension of local fields $\hat{L}_{l} / \hat{K}$, which we denote by $\hat{\mathfrak{f}}_{l}$. Let $\theta$ be the local reciprocity map $\hat{K}^{*} \rightarrow \mathrm{G}\left(\hat{L}_{l} / \hat{K}\right)$. Then $\hat{\mathfrak{f}}_{l}=\mathfrak{p}^{b_{l}}$, where $b_{l}$ is by definition the smallest integer $n$ satisfying $\theta\left(U^{n}\right)=1$. As $\theta$ maps $U^{n}$ onto the $n$th upper ramification group ${ }_{l} G^{n}$ of ${ }_{l} G:=\mathrm{G}\left(\hat{L}_{l} / \hat{K}\right)$, we see that ${ }_{l} G^{b_{l}-1}$ is non-trivial (in fact, ${ }_{l} G^{b_{l}-1}$ is the last non-trivial ramification group in the sense that ${ }_{l} G^{b_{l}-1+\epsilon}=1$ for any $\left.\epsilon>0\right)$. For a discussion of the upper ramification groups, see [10, Chapter 4.3].

By the previous two paragraphs, $l \rightarrow \infty$ implies $a \rightarrow \infty$, which implies $b_{l}-1 \rightarrow$ $\infty$. As $b_{l}-1 \rightarrow \infty$, the largest integral index $c_{l}$ for which the $c_{l}$ th lower ramification group ${ }_{l} G_{c_{l}}$ of ${ }_{l} G$ is non-trivial tends to $\infty$. Now, let $p$ be the rational prime over which $\mathfrak{p}$ lies. We have ${ }_{l} G \leq \mathrm{G}\left(\hat{L}_{l} / \mathbb{Q}_{p}\right):={ }_{l} \Gamma$ and ${ }_{l} G_{n} \leq{ }_{l} \Gamma_{n}$ for any $n$. (It follows from the definition of the ramification groups that ${ }_{l} \Gamma_{e(n+1)-1}={ }_{l} G_{n}$, where $e=e(\mathfrak{p} / p)$.) Therefore, as $l$ increases, the largest $n$ for which ${ }_{l} \Gamma_{n}$ is non-trivial increases as well. From Section 2 we have that the exponent of $p$ in $r d\left(\hat{L}_{l}\right)$ is

$$
\frac{1}{\left|{ }_{l} \Gamma_{0}\right|} \sum_{i=0}^{\infty}\left(\left|{ }_{l} \Gamma_{i}\right|-1\right) \text {. }
$$


The condition of Case \means that ${ }_{l} \Gamma_{0}$ can be bounded independently of $l$. So as $l \rightarrow \infty$, (3) does as well, which in turn implies that $\operatorname{rd}\left(\hat{L}_{l}\right)$, and therefore $\operatorname{rd}\left(L_{l}\right)$, tends to $\infty$, a contradiction.

Case II. There exists $\mathfrak{p} \in S$ such that $\lim \sup e_{L_{n_{l}}}(\mathfrak{p})=\infty$.

Let $\mathfrak{m}=\mathfrak{m}_{0} \mathfrak{m}_{\infty}$ be a modulus of $K$, where $\mathfrak{m}_{0}$ is a product of finite primes and $\mathfrak{m}_{\infty}$ is a product of $r$ real places of $K$. We have the following exact sequence from class field theory:

$$
O^{*} \rightarrow(O / \mathfrak{m})^{*} \rightarrow \mathrm{Cl}_{K}^{\mathfrak{m}} \rightarrow \mathrm{Cl}_{K} \rightarrow 1,
$$

where $O_{K}^{*}$ are the units of the ring of integers $O=O_{K}, \mathrm{Cl}_{K}$ is the ideal class group of $K, \mathrm{Cl}_{K}^{\mathfrak{m}}$ is the ray class group of $K$ modulo $\mathfrak{m}$, and $(O / \mathfrak{m})^{*}$ is defined to be $\left(O / \mathfrak{m}_{0}\right)^{*} \times\{ \pm 1\}^{r}$.

Let $p$ be the rational prime lying below $\mathfrak{p}$. Because $L_{l} / \mathbb{Q}$ is assumed to be Galois, the assumption of Case I implies that $\lim \sup e_{L_{n_{l}}}\left(\mathfrak{p}^{\prime}\right)=\infty$ for every prime $\mathfrak{p}^{\prime}$ of $K$ with $\mathfrak{p}^{\prime} \cap \mathbb{Z}=(p)$. For any rational prime $q$ lying below a prime of $S$, define $\tilde{q}=\prod_{\mathfrak{q} \in S, \mathfrak{q} \mid q} \mathfrak{q}$. For any modulus $\mathfrak{m}$, let $R_{\mathfrak{m}}$ denote the ray class field of $K$ modulo $\mathfrak{m}$. Let $\mathfrak{n}$ be the modulus $\prod_{\mathfrak{q} \in S} \mathfrak{q}$ (note that whether a prime of $K$ is contained in $S$ depends only on the rational prime over which it lies). $L_{l}$ is contained in $R_{\mathfrak{n}^{s(l)}}$ for some positive integer $s(l)$. Our intermediate goal is to show

$$
\limsup _{l \rightarrow \infty}\left[L_{l} \cap R_{\tilde{p}^{s(l)}}: K\right]=\infty .
$$

Notation. Suppose $\left\{E_{l} / F_{l}\right\}_{l}$ is a set of number field extensions indexed by $l$ with $K \subseteq F_{l}$ for all $l$. We say $E_{l} / F_{l}$ is $(*)$ if the ramification above $\mathfrak{p}$ of $E_{l} / F_{l}$ is bounded independently of $l$.

Because $\lim \sup e_{L_{l}}(\mathfrak{p})=\infty$, to prove our intermediate goal, it suffices to show that $L_{l} / L_{l} \cap R_{\tilde{p}^{s(l)}}$ is $(*)$. Let $T$ be the set consisting of the rational primes $\leq \sqrt{N}$ and the infinite real place of $\mathbb{Q}$, i.e., the set of rational primes below the primes of $S$. Consider the field diagram below. It follows from the exact sequence (44) that the $p$-part of $\left[\prod_{q \in T} R_{\tilde{q}^{s(l)}}: R_{\tilde{p}^{s(l)}}\right]$, and therefore of $\left[L_{l} \cap \prod_{q \in T} R_{\tilde{q}^{s(l)}}: L_{l} \cap R_{\tilde{p}^{s(l)}}\right]$, is bounded independently of $l$. All the ramification above $\mathfrak{p}$ in $\prod_{q \in T} R_{\tilde{q}^{s(l)}} / L_{l} \cap R_{\tilde{p}^{s(l)}}$ takes place in $R_{\tilde{p}^{s(l)}} / L_{l} \cap R_{\tilde{p}^{s(l)}}$, and by (44), the prime-to- $p$ part of $e_{R_{\tilde{p}^{s}(l)}}(\mathfrak{p})$ is bounded independently of $l$. Therefore $L_{l} \cap \prod_{q \in T} R_{\tilde{q}^{s(l)}} / L_{l} \cap R_{\tilde{p}^{s(l)}}$ is (*). Thus it suffices to show that $L_{l} / L_{l} \cap \prod_{q \in T} R_{\tilde{q}^{s(l)}}$ is $(*)$. We accomplish this with the following lemma.

Lemma 1. Using the notation above, $\left[R_{\mathfrak{n}^{s(l)}}: \prod_{q \in T} R_{\tilde{q}^{s(l)}}\right]$ is bounded independently of $l$.

Proof. The general case of the lemma will follow if we can show that for any positive integers $a, b$,

$$
\left[R_{\tilde{p}^{a} \tilde{q}^{b}}: R_{\tilde{p}^{a}} R_{\tilde{q}^{b}}\right] \leq \prod_{\mathfrak{p} \mid p}(N \mathfrak{p}-1) \prod_{\mathfrak{q} \mid q}(N \mathfrak{q}-1),
$$

where $q$ is a finite prime of $T$ distinct from $p$, and the products are over primes of $K$ (if $q$ were the infinite place of $\mathbb{Q}$, one could take $2^{r_{1}}$ for the right-hand side of the inequality, $r_{1}$ being the number of real places of $K$ ). Define the maps

$$
\pi_{\tilde{p} \tilde{q}}: O^{*} \rightarrow(O / \tilde{p} \tilde{q})^{*}, \quad \pi_{\tilde{p}}: O^{*} \rightarrow(O / \tilde{p})^{*}, \quad \pi_{\tilde{q}}: O^{*} \rightarrow(O / \tilde{q})^{*} .
$$




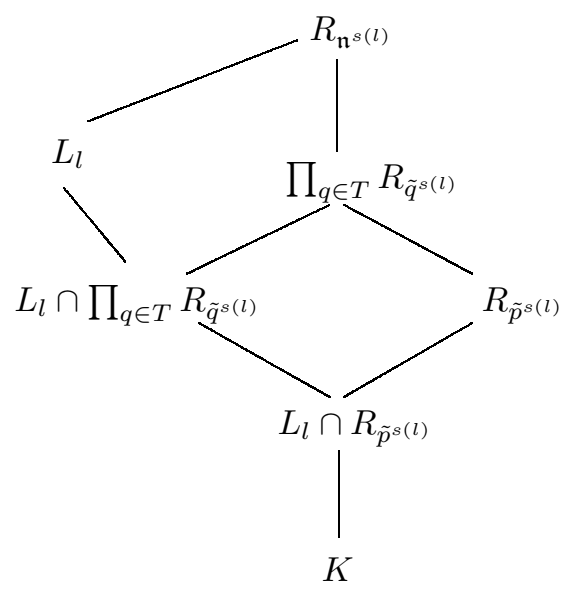

Let $H_{K}$ be the Hilbert class field of $K$. Looking at (41) and using that $R_{\tilde{p}^{a}} \cap R_{\tilde{q}^{b}}=$ $H_{K}$ and that $(O / \tilde{p} \tilde{q})^{*} \cong(O / \tilde{p})^{*} \times(O / \tilde{q})^{*}$, we see that

$$
\left[R_{\tilde{p}^{a} \tilde{q}^{b}}: R_{\tilde{p}^{a}} R_{\tilde{q}^{b}}\right]=\frac{\left|\operatorname{Im}\left(\pi_{\tilde{p}}\right)\right|\left|\operatorname{Im}\left(\pi_{\tilde{q}}\right)\right|}{\left|\operatorname{Im}\left(\pi_{\tilde{p} \tilde{q}}\right)\right|} .
$$

We have a decomposition

$$
\left(O / \tilde{p}^{a}\right)^{*} \cong \prod_{\mathfrak{p} \mid p}(O / \mathfrak{p})^{*} \times\left((1+\mathfrak{p}) /\left(1+\mathfrak{p}^{a}\right)\right) \cong\left(\prod_{\mathfrak{p} \mid p} \mathbb{Z} /(N \mathfrak{p}-1)\right) \times P,
$$

where $P=\prod_{\mathfrak{p} \mid p}\left((1+\mathfrak{p}) /\left(1+\mathfrak{p}^{a}\right)\right)$ is a $p$-group. We have the analogous decomposition of $\left(O / \tilde{q}^{b}\right)^{*}$. Let $H=\operatorname{Im}\left(\pi_{\tilde{p} \tilde{q}}\right)$. Define the natural projections

$$
\begin{gathered}
\phi:(O / \tilde{p} \tilde{q})^{*} \rightarrow(O / \tilde{p})^{*}, \quad \psi:(O / \tilde{p} \tilde{q})^{*} \rightarrow(O / \tilde{q})^{*}, \\
\quad \text { so that } \operatorname{Im}\left(\pi_{\tilde{p}}\right)=\phi(H) \text { and } \operatorname{Im}\left(\pi_{\tilde{q}}\right)=\psi(H) .
\end{gathered}
$$

Since $|\phi(H)|$ and $|\psi(H)|$ both divide $|H|$, we obtain

$$
\frac{|\phi(H)||\psi(H)|}{|H|} \leq \operatorname{gcd}(|\phi(H)|,|\psi(H)|) .
$$

We obtain the result of the lemma by noting that

$$
\operatorname{gcd}(|\phi(H)|,|\psi(H)|)\left|\operatorname{gcd}\left(\left|(O / \tilde{p})^{*}\right|,\left|(O / \tilde{q})^{*}\right|\right)\right| \prod_{\mathfrak{p} \mid p}(N \mathfrak{p}-1) \prod_{\mathfrak{q} \mid q}(N \mathfrak{q}-1) .
$$

This completes the proof of our intermediate goal. With this in hand, we now complete the proof that $X_{N, K}$ is finite under the assumption of Case II.

Set $E_{l}=L_{l} \cap R_{\tilde{p}^{s(l)}}$, and let $\mathfrak{P}_{l}$ be a prime of $E_{l}$ lying above $\mathfrak{p}$. Since $K / \mathbb{Q}$ is assumed to be Galois, the Galois closure of $E_{l} / \mathbb{Q}$ is an abelian extension of $K$ ramified only above $p$, and it is contained in $L_{l}$ because $L_{l} / \mathbb{Q}$ is Galois. But $E_{l}$ is the maximal such field; therefore $E_{l} / \mathbb{Q}$ is Galois. In proving our intermediate goal, we showed that $\lim \sup e_{E_{l}}(\mathfrak{p})=\infty$. Let $\hat{E}_{l}=E_{\mathfrak{P}_{l}}$ and $\hat{K}=K_{\mathfrak{p}}$, and consider the extension of local fields $\hat{E}_{l} / \hat{K}$ with Galois group ${ }_{l} G$. From the structure of $\left(O / \tilde{p}^{s(l)}\right)^{*}$ and the exact sequence (4l) with $\mathfrak{m}=\tilde{p}^{s(l)}$, it follows that there exists $C>0$ such that the prime-to- $p$ part of $\left|{ }_{l} G\right|$ is less than $C$ for all $l$. Let $F_{l}$ be the 
fixed field of the inertia subgroup of $\mathfrak{p}$ of $\mathrm{G}\left(E_{l} / K\right)$ so that $E_{l} / F_{l}$ is totally ramified above $\mathfrak{p}$. Let $\mathfrak{Q}_{l}$ be the prime of $F_{l}$ below $\mathfrak{P}_{l}$ and set $\hat{F}_{l}=F_{l_{\mathfrak{Q}}}$ so that $\hat{E}_{l} / \hat{F}_{l}$ is a totally ramified extension of local fields. Set ${ }_{l} H=\mathrm{G}\left(\hat{E}_{l} / \hat{F}_{l}\right)$. If ${ }_{l} J$ denotes the $p$-Sylow subgroup of ${ }_{l} H$, then $\lim \sup _{l \rightarrow \infty}\left|{ }_{l} J\right|=\infty$. Recall that we are assuming that $L_{l} / K$ is cyclic, so all groups in sight are cyclic. Let $\hat{M}_{l}$ be the fixed field of ${ }_{l} J$, with maximal ideal $\mathfrak{r}_{l}$.
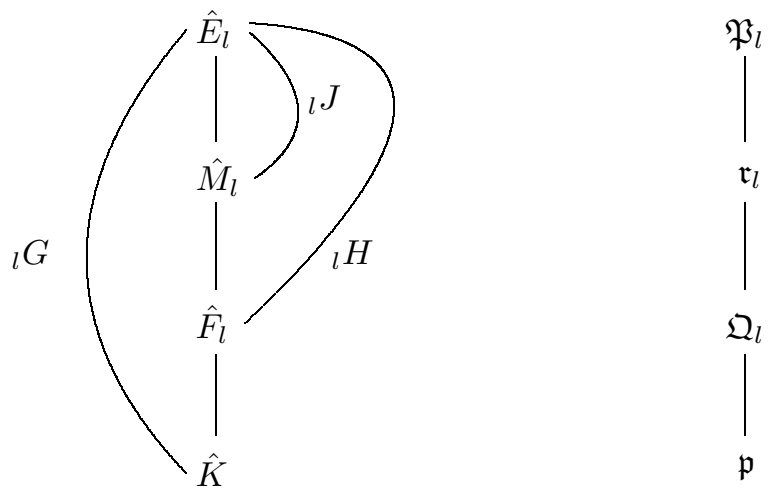

Fix $l$ for the moment and put $\mathfrak{r}=\mathfrak{r}_{l}$. In this paragraph and the next, we summarize a few results from [9] and [10] and apply them to the situation at hand. For non-negative integers $n$, let $U^{n}$ be the $n$-units of $\hat{M}_{l}$, as defined in the proof of Case I. For any real $v \geq 0$, define $U^{v}=U^{n}$, where $n-1<v \leq n$. For all $v \geq 0$, the local reciprocity map $\theta: \hat{M}_{l}{ }^{*} \rightarrow{ }_{l} J$ maps $U^{v}$ onto the upper ramification group ${ }_{l} J^{v}$. It follows that any jumps in the filtration $J^{v}$ must occur at integral values of $v$ (i.e., if $J^{v+\epsilon} \neq J^{v}$ for any $\epsilon>0$, then $v$ is an integer) and that for $v \geq 1$, the quotient ${ }_{l} J^{v} /{ }_{l} J^{v+1}$ of upper ramification groups is isomorphic to a quotient of $U^{v} / U^{v+1}$. For all integrals $n \geq 1$, we have isomorphisms $U^{n} / U^{n+1} \cong \mathfrak{r}^{n} / \mathfrak{r}^{n+1} \cong \mathbb{F}_{\hat{M}_{l}}^{+}$, the last group being the additive group of the residue field of $\hat{M}_{l}$, which is an elementary abelian $p$-group. Since ${ }_{l} J$ is cyclic, we find that for $v \geq 1$, the quotients ${ }_{l} J^{v} /{ }_{l} J^{v+1}$ are either trivial or are cyclic groups of order $p$.

For integral values of $u$, the function $\phi$ satisfying ${ }_{l} J_{u}={ }_{l} J^{\phi(u)}$ is given by

$$
\phi(u)=\left(\frac{1}{\left|{ }_{l} J_{0}\right|} \sum_{i=1}^{u}\left|{ }_{l} J_{i}\right|\right)-1 .
$$

By (5),$\phi(n+1)-\phi(n) \leq 1$, from which it follows that the ${ }_{l} J_{n} /{ }_{l} J_{n+1}$ are also either trivial or cyclic of order $p$. Thus each subgroup of ${ }_{l} J$ occurs as ${ }_{l} J_{n}$ for some $n$. Because jumps in the upper numbering occur at integral values, if $n$ is a nonnegative integer with ${ }_{l} J_{n} \neq{ }_{l} J_{n+1}$, then $\phi(n) \in \mathbb{Z}$. Using this fact along with (5) and the fact that every subgroup of ${ }_{l} J$ occurs as a ramification group, we derive what is essentially the example on p. 76 of [10]:

Lemma 2. For each $i, 0 \leq i \leq t$, there exists $n_{i}$ such that

$$
{ }_{l} J(i)={ }_{l} J_{n_{i}+1}={ }_{l} J_{n_{i}+2}=\cdots={ }_{l} J_{n_{i}+p^{i}},
$$

where $\left|{ }_{l} J\right|=p^{t}$ and ${ }_{l} J(i)$ denotes the unique subgroup of ${ }_{l} J$ of order $p^{t-i}$. Less formally, the subgroup of order $p^{t-i}$ of ${ }_{l} J$ occurs as at least $p^{i}$ lower ramification groups of $l J$. 
Remark 2. - The fact that jumps in the upper ramification groups of ${ }_{l} J^{v}$ occur only at integral values of $v$ also follows from the Hasse-Arf Theorem, which says that if $L / K$ is an abelian extension of local fields with perfect residue fields, then jumps in the upper ramification groups $G^{v}$ of $L / K$ occur only at integral values of $v$ [10, Chapter 5.7].

- One can more directly obtain the fact that ${ }_{l} J_{n} /{ }_{l} J_{n+1}$ is isomorphic to a subgroup of $U^{n} / U^{n+1}$ by considering the map ${ }_{l} J_{n} /{ }_{l} J_{n+1} \hookrightarrow U^{n} / U^{n+1}$ induced by $\sigma \mapsto \sigma(\beta) / \beta$ where $\beta$ is a uniformizer of $\hat{E}_{l}$ [10, Chapter 4.2, Prop. 7].

We use the information just obtained about the ramification groups of ${ }_{l} J$ to obtain bounds for $r d\left(L_{l}\right)$, which will complete the proof of Case II. Since $E_{l} \subseteq L_{l}$, it suffices to show that $\lim _{\sup _{l \rightarrow \infty}} r d\left(E_{l}\right)=\infty$. By Section 2, it then suffices to show that $\lim \sup _{l \rightarrow \infty} r d\left(\hat{E}_{l}\right)=\infty$. The $n$th ramification group of $\hat{E}_{l} / \hat{M}_{l}$ is a subgroup of the $n$th ramification group of $\hat{E}_{l} / \mathbb{Q}_{p}$. By Section 2 , it suffices to show that

$$
\limsup _{l \rightarrow \infty} \frac{1}{\left|{ }_{l} \Gamma_{0}\right|} \sum_{i=0}^{\infty}\left(\left|{ }_{l} \Gamma_{i}\right|-1\right)=\infty,
$$

where ${ }_{l} \Gamma_{n}$ are the ramification groups of $\hat{E}_{l} / \mathbb{Q}_{p}$. Let $\gamma_{n}$ denote the ramification groups of $\hat{E}_{l} / \hat{M}_{l}$. It follows from Lemma 2 that

$$
\frac{1}{\left|\gamma_{0}\right|} \sum_{i=0}^{\infty}\left(\left|\gamma_{l}\right|-1\right) \geq t_{l}-1
$$

where $\left|{ }_{l} J\right|=p^{t_{l}}$. Note that $\lim \sup _{l \rightarrow \infty} t_{l}=\infty$. We also have

$$
\left|{ }_{l} \Gamma_{0}\right|=e\left(\hat{E}_{l} / \hat{F}_{l}\right) e\left(\hat{F}_{l} / \hat{K}\right) e\left(\hat{K} / \mathbb{Q}_{p}\right) .
$$

The quantity $e\left(\hat{K} / \mathbb{Q}_{p}\right)$ is fixed and $e\left(\hat{F}_{l} / \hat{K}\right)=1$. By the definition of ${ }_{l} J$,

$$
\left|{ }_{l} \gamma_{0}\right|=\left|{ }_{l} J\right| \geq \frac{1}{C}\left|\mathrm{G}\left(\hat{E}_{l} / \hat{F}_{l}\right)\right|=\frac{1}{C} e\left(\hat{E}_{l} / \hat{F}_{l}\right) .
$$

(Recall the definition of $C$ from the beginning of the discussion after Lemma 1). Thus we obtain the second inequality in

$$
\frac{1}{\left|{ }_{l} \Gamma_{0}\right|} \sum_{i=0}^{\infty}\left(\left|{ }_{l} \Gamma_{i}\right|-1\right) \geq \frac{1}{\left.\right|_{l} \Gamma_{0} \mid} \sum_{i=0}^{\infty}\left(\left|{ }_{l} \gamma_{i}\right|-1\right) \geq \frac{1}{\left.C\right|_{l} \gamma_{0} \mid e\left(\hat{K} / \mathbb{Q}_{p}\right)} \sum_{i=0}^{\infty}\left(\left|{ }_{l} \gamma_{i}\right|-1\right),
$$

which, using (7), gives the desired result (6), completing Case I.

This completes the proof that $X_{N, K}$ is finite when $K / \mathbb{Q}$ is Galois. We use this to show that $Y_{N, K}$ is finite. We initially maintain the assumption that $K / \mathbb{Q}$ is Galois. Suppose $L \in Y_{N, K}$. We will show there is a constant $A:=A_{N ; K}$, independent of $L$, such that $\operatorname{rd}(\tilde{L})<A_{N ; K}$, where $\tilde{L}$ denotes the Galois closure of $L / \mathbb{Q}$. Suppose we have shown this to be true. The field $\tilde{L}$ is a compositum of abelian extensions of $K$, thus abelian over $K$. The finiteness of $X_{A_{N: K}, K}$ implies that there is some large number field $F_{A}$, which can be chosen independently of $L$, such that $\tilde{L}$, and thus $L$, is contained in $F_{A}$. So, upon showing the existence of $A_{N ; K}$, we will have shown that $Y_{N, K}$ is finite under the assumption that $K / \mathbb{Q}$ is Galois. 

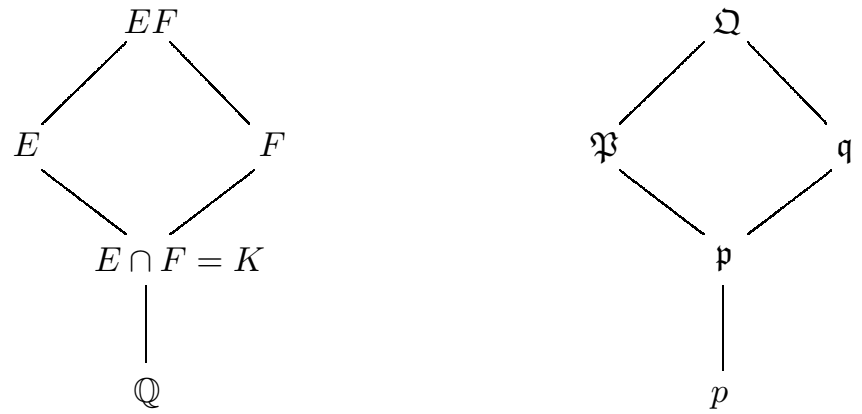

Existence of $A_{N ; K}$ : : For any $\sigma: L \hookrightarrow \mathbb{C}, \sigma$ maps a prime $\mathfrak{P}$ of $L$ ramifying in $L / K$ to a prime $\mathfrak{P}^{\prime}$ of $\sigma(L)$ ramifying in $\sigma(L) / K$, where $\mathfrak{P}$ and $\mathfrak{P}^{\prime}$ lie over the same rational prime $p$. It follows that the rational primes ramifying in $L / \mathbb{Q}$ are the same as those ramifying in $\tilde{L} / \mathbb{Q}$, and using the fact that the discriminant is the norm of the different, one checks that $\operatorname{rd}(L)=\operatorname{rd}(\sigma(L))$. The degree $[L: \mathbb{Q}]$ is the number of embeddings $\sigma: L \hookrightarrow \mathbb{C}$. For each such $\sigma, \tau \sigma(L)=\sigma(L)$ for all embeddings $\tau: \sigma(L) \rightarrow \mathbb{C}$ that fix $K$. There are $[\sigma(L): K]=[L: K]$ such $\tau$, so the number of distinct fields $\sigma(L)$ as $\sigma$ ranges over all embeddings $L \hookrightarrow \mathbb{C}$ is at most $[L: \mathbb{Q}] /[L: K]=[K: \mathbb{Q}]$.

Lemma 3. Let $E$ and $F$ be number fields, both Galois over $E \cap F$. Then

$$
r d(E F) r d(E \cap F) \leq r d(E) r d(F) .
$$

Proof. For notational ease, put $K=E \cap F$. Using (1) from Section 1 multiple times, we have

$$
\begin{aligned}
r d(F) & =N_{K / \mathbb{Q}}\left(d_{F / K}\right)^{\frac{1}{[F: \mathbb{Q}]}} r d(K) \text { and } \\
r d(E F) & =\left(N_{K / \mathbb{Q}} d_{E F / K}\right)^{\frac{1}{[E F: \mathbb{Q}]}} r d(K) \\
& =N_{K / \mathbb{Q}}\left(\left(N_{E / K} d_{E F / E}\right)\left(d_{E / K}^{[E F: E]}\right)\right)^{\frac{1}{[E F: \mathbb{Q}]}} r d(K) \\
& =N_{E / \mathbb{Q}}\left(d_{E F / E}\right)^{\frac{1}{[E F: \mathbb{Q}]}} N_{K / \mathbb{Q}}\left(d_{E / K}\right)^{\frac{1}{[E: \mathbb{Q}]}} r d(K) \\
& =N_{E / \mathbb{Q}}\left(d_{E F / E}\right)^{\frac{1}{[E F: \mathbb{Q}]}} r d(E) .
\end{aligned}
$$

Thus we must show that $N_{E / \mathbb{Q}}\left(d_{E F / E}\right)^{\frac{1}{[E F: \mathbb{Q}]}} \leq N_{K / \mathbb{Q}}\left(d_{F / K}\right)^{\frac{1}{[F: \mathbb{Q}]}}$.

Let $\mathfrak{p}$ be a prime of $K$ above $p$. It suffices to show that the exponent $a$ of $\mathfrak{p}$ in $d_{F / K}^{[E F: F]}$ is greater than or equal to the exponent $b$ of $\mathfrak{p}$ in $N_{E / K}\left(d_{E F / E}\right)$. Let $\mathfrak{q}$ be a prime of $F$ above $\mathfrak{p}$ and $\mathfrak{Q}$ a prime of $E F$ above $\mathfrak{q}$. Let $\mathfrak{P}$ be the prime of $E$ below $\mathfrak{Q}$.

It follows from the definition of ramification groups that the natural restriction map $\mathrm{G}\left(E F_{\mathfrak{Q}} / E_{\mathfrak{P}}\right) \rightarrow \mathrm{G}\left(F_{\mathfrak{q}} / K_{\mathfrak{p}}\right)$ takes $\mathrm{G}_{(\mathfrak{Q} / \mathfrak{P}), i}$ (injectively) into $\mathrm{G}_{(\mathfrak{q} / \mathfrak{p}),\left\lceil\frac{i+1}{e}-1\right\rceil}$, where $\lceil n\rceil$ denotes the least integer greater than or equal to $n$, and $e=e(\mathfrak{Q} / \mathfrak{q})$. From this we find that

$$
\alpha:=\sum_{i=0}\left(\left|\mathrm{G}_{(\mathfrak{Q} / \mathfrak{P}), i}\right|-1\right) \leq e \sum_{i=0}\left(\left|\mathrm{G}_{(\mathfrak{q} / \mathfrak{p}), i}\right|-1\right):=e \gamma .
$$


One checks that

$$
a=\gamma g_{F / K}(\mathfrak{p}) f_{F / K}(\mathfrak{p})[E F: F] \text { and } b=f_{E / K}(\mathfrak{p}) g_{E / K}(\mathfrak{p}) g_{E F / E}(\mathfrak{P}) f_{E F / E}(\mathfrak{P}) \alpha .
$$

We can rewrite $b$ as

$$
\alpha f_{E F / F}(\mathfrak{q}) g_{E F / F}(\mathfrak{q}) f_{F / K}(\mathfrak{p}) g_{F / K}(\mathfrak{p}) .
$$

Using $[E F: F]=e f_{E F / F}(\mathfrak{q}) g_{E F / F}(\mathfrak{q})$ and $\alpha \leq e \gamma$, we find that $b \leq a$, as desired. This completes the proof of the lemma.

For our purposes, we only need the weaker result $r d(E F) \leq r d(E) r d(F)$. Returning to our previous notation, by Lemma 3 and induction on $n$ we obtain

$$
r d\left(\sigma_{1}(L) \cdots \sigma_{n}(L)\right) \leq \prod_{i=1}^{n} r d\left(\sigma\left(L_{i}\right)\right)=r d(L)^{n} .
$$

Thus $r d(\tilde{L}) \leq r d(L)^{[K: \mathbb{Q}]}$, and we may take $A_{N ; K}=N^{[K: \mathbb{Q}]}$.

Thus, as long as $K / \mathbb{Q}$ is Galois, we have shown that $Y_{N, K}$ is finite. We now show that the assumption $K / \mathbb{Q}$ Galois is unnecessary. We have the following theorem from algebraic number theory, suitably modified for our purposes [6, Chapter 3.2, Thm. 2.6].

Theorem 2. Let $E / F$ be a finite Galois extension of number fields of degree $n$ and let $\mathfrak{p}$ be a prime of $F$ lying below a prime $\mathfrak{P}$ of $E$ with ramification index $e=e(\mathfrak{P} / \mathfrak{p})$. Then

$$
v_{\mathfrak{p}}\left(d_{E / F}\right) \leq n\left(1+v_{\mathfrak{p}}(e)-1 / e\right) .
$$

Let $\tilde{K}$ denote the Galois closure of $K / \mathbb{Q}$ and suppose $L / K$ is abelian with $r d(L) \leq N$. We show there is a constant $C_{N, K}$ such that

$$
r d(L \tilde{K}) \leq C_{N, K} r d(L) .
$$

Suppose we know this to be true. Since $\tilde{K} / \mathbb{Q}$ is Galois, there exists a large number field $F_{C_{N, K} N, \tilde{K}}$ such that $E \subseteq F_{C_{N, K} N, \tilde{K}}$ for any $E \in Y_{C_{N, K} N, \tilde{K}}$. In particular, since $L \tilde{K} / \tilde{K}$ is abelian, $L \tilde{K} \in Y_{C_{N, K} N, \tilde{K}}$. Thus

$r d(L) \leq N \Longrightarrow \operatorname{rd}(L \tilde{K}) \leq C_{N, K} N \Longrightarrow L \subseteq L \tilde{K} \subseteq F_{C_{N, K} N, \tilde{K}} \Longrightarrow Y_{N, K}$ is finite.

We show that such a $C_{N, K}$ exists. It follows from (1) that

$$
r d(L \tilde{K})=\left(N_{L / \mathbb{Q}} d_{L \tilde{K} / L}\right)^{\frac{1}{[L \tilde{K}: \mathbb{Q}]}} r d(L) .
$$

The primes ramifying in $\tilde{K} / K$ are fixed with $K$, so there exists a finite set of rational primes $R$, independent of $L$, such that every prime of $L$ ramifying in $L \tilde{K} / L$ lies above a prime in $R$. Let $\mathfrak{q}$ be a prime of $L$ ramifying in $L \tilde{K} / L$ lying above a rational prime $q$.

Let $e=e_{L \tilde{K}}(\mathfrak{q})$ (note that $L \tilde{K} / L$ is Galois). By Theorem 2 the exponent of $\mathfrak{q}$ in $d_{L \tilde{K} / L}$ is at most

$$
[L \tilde{K}: L]\left(1+v_{\mathfrak{q}}(e)-1 / e\right) \leq[L \tilde{K}: L]\left(1+v_{\mathfrak{q}}(e)\right) .
$$




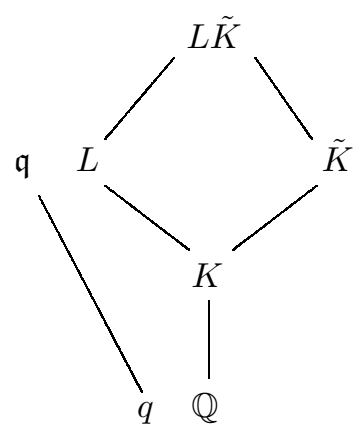

We have the analogous bound for every prime $\mathfrak{q}$ above $q$ in $L$. Let $e_{\mathfrak{q}}=e_{L / \mathbb{Q}}(\mathfrak{q})$ and $f_{\mathfrak{q}}=f_{L / \mathbb{Q}}(\mathfrak{q})$ (do not confuse $e$ with $e_{\mathfrak{q}}$ ). We obtain

$$
\begin{aligned}
v_{q}\left(N_{L / \mathbb{Q}} d_{L \tilde{K} / L}\right) & \leq \sum_{\mathfrak{q} \mid q}[L \tilde{K}: L] f_{\mathfrak{q}}\left(v_{\mathfrak{q}}(e)+1\right) \\
& =[L \tilde{K}: L] \sum_{\mathfrak{q} \mid q} f_{\mathfrak{q}}\left(e_{\mathfrak{q}} v_{q}(e)+1\right) \\
& \leq[L \tilde{K}: L][L: \mathbb{Q}][L \tilde{K}: L],
\end{aligned}
$$

where we have used the fact that $\sum f_{\mathfrak{q}} e_{\mathfrak{q}}=[L: \mathbb{Q}]$ and the obvious inequality $v_{q}(e)+1 \leq[L \tilde{K}: L]$ to obtain the final inequality above. In light of (8), taking

$$
C_{N, K}=\prod_{q \in R} q^{[\tilde{K}: K]}
$$

gives the result.

When $n=1$, this completes the proof of Theorem 1 in its entirety. We obtain the theorem for general $n$ by induction:

Let $L \in Y_{n, N, K}$. Let $G^{(i)}$ denote the $i$ th derived subgroup of $G$, so $n$ is the smallest integer with $G^{n}=1$. The quotient $G / G^{n-1}$ corresponds to an intermediate field $L_{0}$ of $L / K$ with $G / G^{n-1} \cong \mathrm{G}\left(L_{0} / K\right) . L_{0} \subseteq L$ implies $\operatorname{rd}\left(L_{0}\right) \leq \operatorname{rd}(L)$, so $L_{0} \in Y_{n-1, N, K}$. By induction there are only a finite number of possibilities for $L_{0}$. For each such $L_{0}, \mathrm{G}\left(L / L_{0}\right)=G^{n-1}$ is abelian, so by the $n=1$ case of Theorem 1 applied to $L_{0}$ and $N$, there are only finitely many possibilities for $L$.

Remark 3. The proof of Theorem[1]does not lend itself to an effective bound of the size of $Y_{n, N, K}$ in terms of $n, N$ and $K$.

Corollary 1. Fix $N>0$, a positive integer $n$, and a number field $K$. Then

$$
\#\left\{L: L / K \text { is solvable, } \mathrm{G}(L / K) \subseteq \mathrm{GL}_{n}(\mathbb{F}) \text { and } \operatorname{rd}(L) \leq N\right\}
$$

is finite, where $\mathbb{F}$ is a finite field that is allowed to vary with $L$.

Proof. It is a theorem of Zassenhaus 12 that the length of any solvable subgroup of $G L_{n}(\mathbb{F})$ has a finite bound depending on $n$, independent of $\mathbb{F}$ (in fact $\mathbb{F}$ need not even be finite). The result now follows from Theorem 1 


\section{Further Questions}

As mentioned in the introduction, a natural extension of Theorem 1 is to consider the size of various subsets of $Z_{N, K}$. Kedlaya [4, generalizing work by Yamamoto [13, has shown that for any $n$, there exist infinitely many real quadratic number fields $K$ admitting an unramified degree $n$ extension $L$ with $G(\tilde{L} / K)$ isomorphic to the alternating group $A_{n}$, where $\tilde{L}$ denotes the Galois closure of $L / K$. It is unknown (at least to the author), however, whether or not a fixed (say real quadratic) number field may admit an unramified degree $n$ extension with Galois closure having Galois group $A_{n}$ for infinitely many $n$.

Fix a number field $K$. Suppose that $Z_{N, K}$ is infinite. One can ask whether $Z_{N, K}$ must contain an infinite class field tower. Maire [5] has proven the existence of infinite unramified extensions of number fields with class number one. Let $K$ be such a number field with infinite degree unramified extension $L$. Maire constructs $L$ by constructing an infinite class field tower of a finite unramified extension of $K$. This leaves open the question of whether a general infinite unramified extension $L / K$ must contain an infinite class field tower, i.e., whether $G(L / K)$ must have a pro-solvable subquotient. One can also ask the same question but replace the condition $L / K$ unramified with the condition $\operatorname{rd}(L)<N$ (meaning $r d(M)<N$ for every field $M$ between $L$ and $K$ with $M / K$ finite). The answers to such questions are likely beyond the scope of class field theory.

Since there are only finitely many number fields of bounded root discriminant of any fixed degree, finding infinite subsets of $Z_{N, K}$ amounts to finding $L \in Z_{N, K}$ with arbitrarily large degree. One may alternatively consider how many number fields $L$ of degree $n$ over $K$ with $r d(L)<N$ exist, with $n$ fixed and $N$ varying. Significant work in this direction has been done by Ellenberg and Venkatesh [1] and others.

\section{ACKNOWLEDGMENTS}

The author would like to thank his advisor, Joe Silverman, for suggesting the question of the finiteness of $Y_{N, K}$ and for his helpful conversations. He would also like to thank the referee for helpful comments.

\section{REFERENCES}

1. Jordan S. Ellenberg and Akshay Venkatesh, The number of extensions of a number field with fixed degree and bounded discriminant, Ann. of Math. (2) 163 (2006), no. 2, 723-741. MR2199231 (2006j:11159)

2. Farshid Hajir and Christian Maire, Tamely ramified towers and discriminant bounds for number fields. II, J. Symbolic Comput. 33 (2002), no. 4, 415-423. MR1890578(2003h:11137)

3. David Hilbert, The theory of algebraic number fields, Springer-Verlag, Berlin, 1998. Translated from the German and with a preface by Iain T. Adamson and with an introduction by Franz Lemmermeyer and Norbert Schappacher. MR1646901 (99j:01027)

4. Kiran Kedlaya, A construction of polynomials with squarefree discriminants, Proc. Amer. Math. Soc. 140 (2012), no. 9, 3025-3033. MR2917075

5. Christian Maire, On infinite unramified extensions, Pacific J. Math. 192 (2000), no. 1, 135142. MR 1741025 (2000j:11160)

6. Jürgen Neukirch, Algebraic number theory, Grundlehren der Mathematischen Wissenschaften [Fundamental Principles of Mathematical Sciences], vol. 322, Springer-Verlag, Berlin, 1999. Translated from the 1992 German original and with a note by Norbert Schappacher, with a foreword by G. Harder. MR.1697859 (2000m:11104) 
7. A. M. Odlyzko, Bounds for discriminants and related estimates for class numbers, regulators and zeros of zeta functions: a survey of recent results, Sém. Théor. Nombres Bordeaux (2) 2 (1990), no. 1, 119-141. MR1061762 (91i:11154)

8. Peter Roquette, On class field towers, Algebraic Number Theory (Proc. Instructional Conf., Brighton, 1965), Thompson, Washington, D.C., 1967, pp. 231-249. MR0218331 (36:1418)

9. J.-P. Serre, Local class field theory, Algebraic Number Theory (Proc. Instructional Conf., Brighton, 1965), Thompson, Washington, D.C., 1967, pp. 128-161. MR0220701 (36:3753)

10. Jean-Pierre Serre, Local fields, Graduate Texts in Mathematics, vol. 67, Springer-Verlag, New York, 1979. Translated from the French by Marvin Jay Greenberg. MR554237 (82e:12016)

11. Joseph H. Silverman, The arithmetic of elliptic curves, second ed., Graduate Texts in Mathematics, vol. 106, Springer, Dordrecht, 2009. MR2514094(2010i:11005)

12. D. A. Suprunenko, Matrix groups, Translations of Mathematical Monographs, Vol. 45, American Mathematical Society, Providence, R.I., 1976. Translated from the Russian, translation edited by K. A. Hirsch. MR0390025 (52:10852)

13. Yoshihiko Yamamoto, On unramified Galois extensions of quadratic number fields, Osaka J. Math. 7 (1970), 57-76. MR0266898(42:1800)

Department of Mathematics, Brown University, 151 Thayer Street, Providence, RHODE ISLAND 02912

E-mail address: JLeshin@math.brown.edu 